ERRATUM

\title{
ORGANOGENESIS OF LYMPHOID TISSUES
}

\section{Reina E. Mebius}

Nature Reviews Immunology 3, 292-303 (2003)

In Figure 5, on page 299, two items of text were incorrectly placed in the figure so that they did not convey the author's meaning. The label "IL-7R $\rightarrow$ LT" should have been placed in part $\mathrm{b}$ of the figure above the arrow in the lower left-hand corner of the figure. The label "IL-7R, CXCR5, TRANCER $\rightarrow$ LT" should have been placed above the arrow in the lower right-hand corner of the figure.

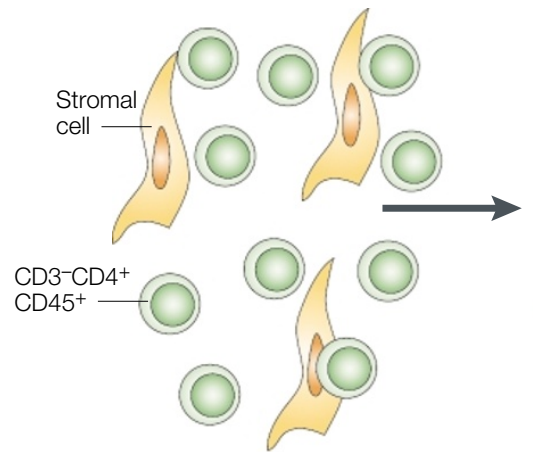

E12.5-E14.5 Brachial lymph nodes Axillary lymph nodes

b

E14.5-birth

Inguinal lymph nodes

Popliteal lymph nodes

Peyer's patches

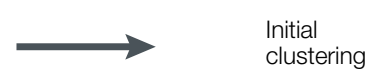

$\mathrm{LL}-7 \mathrm{R} \rightarrow \mathrm{LT}$

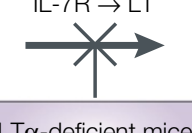

Initial

clustering

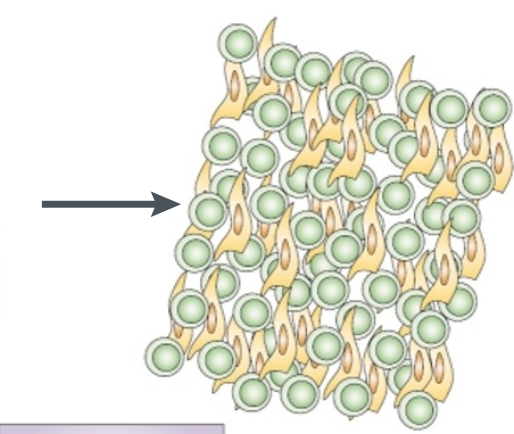

LT $\alpha$-deficient mice

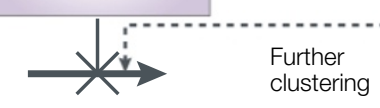

IL-7R, CXCR5 TRANCER $\rightarrow$ LT

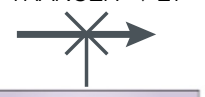

Further

clustering

LT $\alpha$-deficient mice

Figure 5 | Model of increasing dependency on $\mathbf{L T} \alpha_{1} \beta_{2}$ for the initial cell clustering. a | For brachial and axillary lymph nodes, which develop early during embryogenesis, the initial cell clustering might occur in the absence of lymphotoxin (LT) $\alpha$. Therefore, small cell clusters can form in LT $\alpha$-deficient mice, and after injection of an agonistic monoclonal antibody specific for lymphotoxin- $\beta$ receptor (LT $\beta R$ ), these clusters further develop into larger clusters. Following initial clustering, expression of $L T \alpha_{1} \beta_{2}$ is required for the further accumulation of cells. $\mathbf{b} \mid$ The first clustering of cells for the formation of lymphoid organs that develop later in gestation - that is, inguinal and popliteal lymph nodes and Peyer's patches - requires the expression of $L T \alpha$. As no cell clusters can form at these locations in LT $\alpha$-deficient mice, induction of signalling through LT $\beta R$ does not result in further formation of large clusters. Once the first small cell clusters are formed, expression of $L T \alpha_{1} \beta_{2}$ is still required for the further accumulation of cells.

\section{ERRATUM}

\section{REGULATORY T CELLS UNDER SCRUTINY}

Jean François Bach

Nature Reviews Immunology 3, 189-198 (2003)

The author's name was presented incorrectly: the correct format is Jean-François Bach. The PubMed record for this article will be corrected. 